\title{
Energy efficient information collection in wireless sensor networks using adaptive compressive sensing
}

\author{
Chun Tung Chou ${ }^{1}$, Rajib Rana ${ }^{1}$, Wen $\mathrm{Hu}^{2}$ \\ ${ }^{1}$ School of Computer Science and Engineering, University of New South Wales (UNSW), Sydney, Australia \\ ${ }^{2}$ Autonomous Systems Laboratory, CSIRO ICT Centre, Brisbane, Australia \\ Email: ctchou@cse.unsw.edu.au; rajibr@cse.unsw.edu.au; Wen.Hu@csiro.au
}

\begin{abstract}
We consider the problem of using Wireless Sensor Networks (WSNs) to measure the temporal-spatial field of some scalar physical quantities. Our goal is to obtain a sufficiently accurate approximation of the temporal-spatial field with as little energy as possible. We propose an adaptive algorithm, based on the recently developed theory of adaptive compressive sensing, to collect information from WSNs in an energy efficient manner. The key idea of the algorithm is to perform "projections" iteratively to maximise the amount of information gain per energy expenditure. We prove that this maximisation problem is NP. hard and propose a number of heuristics to solve this problem. We evaluate the performance of our proposed algorithms using data from both simulation and an outdoor WSN testbed. The results show that our proposed algorithms are able to give a more accurate approximation of the temporal-spatial field for a given energy expenditure.
\end{abstract}

\section{INTRODUCTION}

We consider the problem of using Wireless Sensor Networks (WSNs) to measure the temporal-spatial field of some scalar physical quantities, e.g. the variation of temperature over a certain geographical area over a certain time. One method of measuring such a temporal-spatial field (or data field for short) is to have all the sensors in the WSNs return their measurements to a sink (or data fusion centre) at regular time intervals. This method gives the maximum amount of information on the data field but at the same time requires the maximum amount of energy to collect the information. Instead, it may be possible to use a reduced amount of energy to obtain a sufficiently accurate approximation of the data field. This is possible if the sensor measurements are correlated, which happens when the sensors are densely deployed. This paper proposes an adaptive algorithm to obtain a sufficiently accurate approximation of the data field with as little energy as possible. Although there is a rich literature on adaptive sampling for WSNs, e.g. [6], [11], [13], [16], to the best of our knowledge, our algorithm is the first one that uses adaptive compressive sensing.

Compressive sensing [2], [3], [7] is a collection of recently proposed sampling methods in Information Theory. The promise of compressive sensing is that it can obtain a sufficiently accurate approximation of an unknown data field by using a small number of generalised measurements, which are known as projections in the compressive sensing literature. (The concept of projections will be explained in Section II.) In adaptive compressive sensing [10], [20], these projections are iteratively computed in order to extract the maximum amount of information from the unknown data field with as few projections as possible.

Although the existing adaptive compressive sensing algorithms can obtain good approximation of the data field with a smaller number of projections than their non-adaptive counterparts, these algorithms cannot be directly applied to WSNs because they do not take into consideration the energy required to acquire a projection in WSNs. For data collection in WSNs, the goal is to collect as much information with as little energy (rather than as few projections) as possible. In this paper, we propose a method to iteratively compute projections that maximises the ratio of information gain to the energy required to acquire the information in order to realise energy-efficient information collection in WSNs (Sections II and III). We show that this maximisation problem is NP-hard and propose a number of heuristics to solve this problem (Section III). We evaluate the performance of our proposed algorithms using simulation data and data collected from an outdoor WSN (Section IV). Our evaluation shows that our proposed algorithm gives a better accuracy for a given energy expenditure.

\section{INFORMATION COLLECTION FRAMEWORK}

We model the WSN as a graph $G=(\{s\} \cup V, E)$ where $s$ is the sink node, $V=\{1,2, \ldots, n\}$ is the set of sensor nodes and $E$ is the set of edges where an edge exists between two sensor nodes if they are within the communication range of each other. (Note that the framework described here can equally be applied to a cluster with $n$ sensor nodes and a cluster head, therefore the method is scalable.) We assume that the sensors are synchronised. We consider a snapshot of the temporal-spatial field where at a particular time $t$, the sensors make a measurement. Let the noise-free sensor reading of sensor node $i$ (where $i=1, \ldots, n$ ) be $x_{i}$. The actual (noisy or measured) sensor reading is assumed to be corrupted by an independent and identically distributed zero mean Gaussian noise of variance $\sigma^{2}$. Let the sensor noise at sensor $i$ be $e_{i}$, then the actual sensor reading at sensor node $i$ is $y_{i}=x_{i}+e_{i}$. We will use $x$ to denote the vector $\left[x_{1}, x_{2}, \ldots, x_{n}\right]^{T}$ where $T$ denotes matrix transpose; the vectors $e$ and $y$ are similarly defined. Our goal is to obtain an approximation $\hat{x}=\left[\hat{x}_{1}, \hat{x}_{2}, \ldots, \hat{x}_{n}\right]^{T}$ of the true data field $x$. We will measure the accuracy of the approximate data field by using the relative error $\frac{\|x-\hat{x}\|}{\|x\|}$ where $\|x\|=\sqrt{\sum_{i=1}^{n} x_{i}^{2}}$ denotes the $2-$ norm of $x$. 
We assume that both sensing energy and energy required for computation is negligible, which are fairly typical assumptions in WSNs [9]. The energy consumption in the WSNs is dominated by radio communications in transmitting and receiving data packets. In this paper, we will measure the energy consumption by the total number of transmissions required to collect the information on data field. The reference scenario is when all nodes in the network send the data to the sink which requires a number of transmissions of the order of $n^{2}$ in the multi-hop scenario. This work distinguishes itself from other works in energy efficient adaptive sensing, e.g. [6], [11], [13], [16], in that it uses recent works in adaptive compressive sensing [10], [20]. However, existing work in adaptive compressive sensing only takes into consideration the accuracy of the approximate data field. In order to apply adaptive compressive sensing to WSNs, our work in this paper takes both accuracy and energy into consideration. In particular, we will show that there is a "cross-layer" interaction in using adaptive compressive sensing in WSNs where one needs to take both accuracy (at the application layer) and routing into consideration. In order to understand this "cross-layer" interaction, we will need to understand what a projection is in compressive sensing.

\section{A. Projections in compressive sensing}

A distinctive feature of compressive sensing is that it uses projections to collect information. For a snapshot of the noisy data field $\left\{y_{i}\right\}$, the projection of the vector $y$ on a projection vector $p=\left[p_{1}, p_{2}, \ldots, p_{n}\right]^{T}$ is defined by the inner product $p^{T} y=\sum_{i=1}^{n} p_{i} y_{i}$. Let us illustrate the concept of projection vectors and how projections can be calculated in a WSN with a few examples. Consider the network shown in Figure 1 with 4 sensor nodes $\{1,2,3,4\}$ and sink node $s$.

Example 1: If the projection vector $p$ is $[0.2,0.3,0.4,0.1]$, then the projected value $p^{T} y=0.2 y_{1}+0.3 y_{2}+0.4 y_{3}+0.1 y_{4}$. The sink can obtain this projected value without the sensors sending their sensor readings to the sink. This can be achieved by the sink passing a message along the tour $S-1-2-3-4-S$ using source routing in the WSN. The message contains the entire projection vector $p$ as well as a field in the message to store the intermediate result of the projection calculation. As the message travels through the tour, each sensor computes its contribution to the projected value and adds it to the intermediate result. After that, the sensor writes the new intermediate result to the message and forwards the message to the next hop. For example, sensor node 2 will receive from sensor node 1 a message with $0.2 y_{1}$ as the intermediate result; sensor 2 will compute $0.3 y_{2}$ and add this to $0.2 y_{1}$, then it will write the sum to the message and then pass it on to the next hop. Note that the computation of this projection requires 5 wireless transmissions.

Example 2: If the projection vector $p$ is $[0.1,0.2,0,0]^{T}$, then the projected value $p^{T} y=0.1 y_{1}+0.2 y_{2}$. This can be computed by passing a message along the tour $S-1-2-1-S$ since sensor readings from sensors 3 and 4 are not needed to compute this projection. Therefore, the calculation of this projection requires only 4 wireless transmissions. Note that

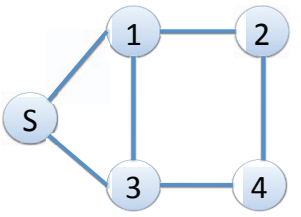

Fig. 1. Example WSN.

in general, the calculation of a projection only requires a message to be passed along those sensor nodes with a nonzero projection vector coefficient. From an energy efficiency point of view, one would therefore aim to find the shortest tour (or aggregation tree) which passes through all sensor node required.

Example 3: If the projection vector $p$ is $[0,0,1,0]^{T}$, then the projected value $p^{T} y=y_{3}$. Therefore, this projection vector corresponds to collecting the noisy sensor reading from sensor 3 . This example aims to show that a projection is a general method of collecting data and collecting a sensor reading from a sensor is in fact a special case of performing a projection.

\section{B. Adaptive compressive sensing for WSNs}

With the above example, we have explained what a projection is and how a projection can be computed in WSN. Assuming that the sink has the projection vectors $\phi_{j}(j=1, \ldots, k)$ and their corresponding projected values $z_{j}=\phi_{j}^{T} y(j=1, \ldots, k)$, then the sink can use compressive sensing to estimate the noise free data field $x$. Most of the compressive sensing algorithms proposed to date are non-adapative which means the projection vectors are not chosen according to information collected so far. Recent effort in adaptive compressive sensing [10], [20] shows that by choosing the coefficients in the projection vector to maximise the information content, it is possible to collect more information (or achieve a smaller relative error in estimating $x$ ) using a smaller number of projections. In this paper, we will use the generic adaptive compressive sensing algorithm outlined under Algorithm 0.

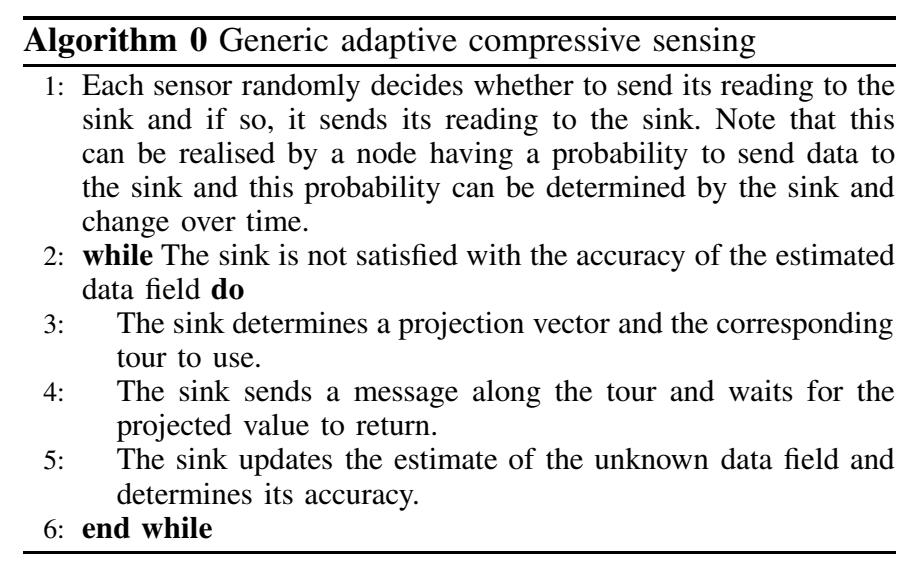

The general idea of the generic adaptive compressive sensing algorithm is as follows. In line 1, a small number of sensors 
randomly decide to return their sensor readings to the sink. These random samples will allow the sink to estimate the data field and decide whether it needs to use the iteration in lines 2-6 to collect further information from the sensor field. This iteration continues until the sink is satisfied with the accuracy of the estimated data field. Note that the rationale behind the above adaptive compressive sensing is the same as that of optimal experiment design in statistics [5] and active learning in machine learning [14].

A key step in the above algorithm is line 3 where a good projection vector has to be determined. This step is also studied in the adaptive compressive sensing algorithms in [10], [20] where a projection vector which maximises the amount of information gain is determined. However, these earlier works are not suitable for WSNs because they do not take into consideration the energy required to acquire a projection. We show in our earlier examples that the energy required to acquire a projection depends on the length of the tour (or the size of the aggregation tree) needed to obtain the projection, which is in turn a function of the locations of the non-zero coefficients in the projection vector. Therefore, the choice of the coefficient of a projection vector can affect both the information content and the energy expenses (via the choice of route to obtain the projection). This means that a projection vector should be chosen to collect as much information on the unknown data field with as little energy as possible, which is a design problem requiring information from both the application and routing layers. In Section III, we propose an optimisation problem to find a projection vector which maximises the information gain per energy expenditure and we prove that this optimisation problem is NP-hard. Because of NP-hardness of the problem, we will propose a number of heuristics in Section III to solve this problem.

We will measure the energy consumption in WSNs by counting the number of packet transmissions, e.g. a node which is $k$ hops away from the sink will require $k$ packet transmissions to reach the sink. In line 1 of Algorithm 0 , energy is consumed to send packets to the sink, while in lines $2-6$, energy is consumed to send packets along the tour. It can be seen that if a good probability can be found in line 1 , this can minimise the energy consumption in lines 2-6. A possibility is to determine this probability from past history of the data. In this paper, we assume a reasonable estimate of this probability can be obtained and focus on the design of a projection vector which balances information gain and energy consumption. We would also like to remark that it is possible to modify line 1 to have a random number of sensors initiating a number of random projections towards the sink, this will improve the information content at the sink and will be studied in the future. We also assume that the sink (or cluster head) knows the topology of the network (or cluster).

\section{ADAPTIVE PROJECTION VECTORS}

In this section, we show how a projection vector can be computed to provide a high information gain on the unknown data field with small energy expenditure, i.e. line 3 of Algorithm 0. In Section III-A, we derive an expression on the expected information gain for a projection vector. We then formulate an optimisation problem in Section III-B which balances information gain against energy expenditure and show that the problem is NP-hard. In Section III-C, we will present a number of heuristics to solve the proposed optimisation problem.

\section{A. Expected information gain of a projection vector}

In this section, we derive an expression for the expected information gain of a projection vector. We assume that $k$ projections have already been measured over the data field. Let $\phi_{1}, \phi_{2}, \ldots, \phi_{k} \in \mathbb{R}^{n}$ denote these $k$ projection vectors and $z_{1}, \ldots, z_{k}$ denote the corresponding projected values, i.e. $z_{i}=\phi_{i}^{T} y$. The advantage of compressive sensing is that it can estimate $x$ even if $k$ is less than $n$ (= number of sensors $=$ number of data points in a snapshot) provided that we know that the noise-free data field vector $x$ is compressible [1], [2].

A vector $x$ is said to be compressible in a basis [19] $B \in \mathbb{R}^{n \times n}$ (where the basis vectors are the columns of $B$ ) if the coefficients $w=B^{-1} x$ of $x$ in the basis $B$ has the following property: the $\ell$-th largest (in absolute value) coefficient of $w$ decays faster than $\ell^{-\frac{1}{\beta}}$ for some $\beta \in(0,1)$ [1]. Intuitively, a signal is compressible if the signal contains redundancy. Therefore, for a sensor data field whose data is correlated, it is reasonable to assume that the signal is compressible. In this paper, we will make the assumption that we know a priori the basis in which the signal is compressible. This is by no means a restriction as preliminary experiments can be carried out to determine this basis.

Assuming that the noise-free data field $x$ is compressible in the basis $B$ and $x=B w$ where $w$ are the coefficients of $x$ in the basis $B$. We can write the projections using the following data equation:

$$
\underbrace{\left[\begin{array}{c}
z_{1} \\
\vdots \\
z_{k}
\end{array}\right]}_{z}=\underbrace{\left[\begin{array}{c}
\phi_{1}^{T} \\
\vdots \\
\phi_{k}^{T}
\end{array}\right]}_{\Phi} y=\Phi(x+e)=\Phi B w+\Phi e
$$

Note that most compressive sensing algorithms assume that the noise on the projected values are independent. However, since a noisy sensor reading may appear in multiple projections in Eq. (1), the noise on the projected values are correlated. In order to de-correlate the noise in the projected values, we compute the Cholesky factorisation [8] of $\Phi \Phi^{T}=R^{T} R$ and pre-multiply Eq. (1) with $R^{-T}$ to obtain the revised data equation:

$$
\underbrace{R^{-T} z}_{\tilde{z}}=\underbrace{R^{-T} \Phi B}_{\tilde{\Phi}} w+R^{-T} \Phi e
$$

We can now input $\tilde{\Phi}$ and $\tilde{z}$ to the Bayesian compressive sensing (BCS) algorithm [10] which returns the posteriori probability distribution of the estimate of unknown vector $w$ and unknown noise variance $\sigma^{2}$. Let us denote the estimate of $w$ by $\hat{w}$. The probability distribution of $\hat{w}$ is Gaussian with mean $\mu \in \mathbb{R}^{n}$ and covariance matrix $\Sigma \in \mathbb{R}^{n \times n}$. (Note that $\mu$ and $\Sigma$ are the outputs of the BCS algorithm and are therefore 
functions of $\tilde{\Phi}$ and $\tilde{z}$.) We can now obtain $\hat{x}=B \hat{w}$. Therefore, the estimate of the data field $\hat{x}$ is Gaussian distributed with mean $B \mu$ and covariance $B \Sigma B^{T}$. Lastly, we will use $\hat{\sigma}^{2}$ to denote the posteriori estimate of the measurement noise variance $\sigma^{2}$

Since our goal is to obtain a sufficiently accurate estimate of the unknown data field, this is equivalent to minimising the uncertainty in the estimate $\hat{x}$. Since $\hat{x}$ is given by a continuous probability distribution, we can measure the uncertainty in $\hat{x}$ by using the differential entropy of $\hat{x}$ where a small differential entropy implies a small uncertainty. Thus, we can measure the additional information provided by a new projection vector $p$ by the reduction in differential entropy in the estimate $\hat{x}$ caused by using $p$ together with $\left\{\phi_{i}\right\}_{i=1, \ldots, k}$. The use of the new data equation (2) means that the results in [10] on the entropy reduction by an additional projection vector is no longer applicable. We have the following new result.

Lemma 1: The reduction of differential entropy $\Delta H$ in the estimate of the unknown data field by using an additional projection vector $p \in \mathcal{B} \cap\left\{p \in \mathbb{R}^{n \times 1}: V^{T} p \neq 0\right\}$ is given by

$$
\Delta H(p)=\frac{1}{2} \log \left(1+\frac{1}{\hat{\sigma}^{2}} \frac{p^{T} V V^{T} B \Sigma B^{T} V V^{T} p}{p^{T} V V^{T} p}\right)
$$

where $\mathcal{B}=\left\{p \in \mathbb{R}^{n \times 1}:\|p\|=1\right\}$, and, $V$ satisfy $\Phi V=0$ and $V^{T} V=I$ where $I$ denotes the identity matrix. (Note that $V$ is in fact an orthonormal basis of the null space of $\Phi$.)

Proof: Using the same method in [10], it can be shown that the reduction of entropy by using an additional projection vector $p$ when the data equation is given as in equation (2) is:

$$
\begin{aligned}
\Delta H(p)= & \frac{1}{2} \log \operatorname{det}\left(A+\frac{1}{\hat{\sigma}^{2}} B^{T} \breve{\Phi}^{T}\left(\breve{\Phi} \breve{\Phi}^{T}\right)^{-1} \breve{\Phi} B\right)- \\
& \frac{1}{2} \log \operatorname{det}\left(A+\frac{1}{\hat{\sigma}^{2}} B^{T} \Phi^{T}\left(\Phi \Phi^{T}\right)^{-1} \Phi B\right)
\end{aligned}
$$

where det is the determinant of a matrix and

$$
\breve{\Phi}=\left[\begin{array}{c}
\Phi \\
p^{T}
\end{array}\right] \text {. }
$$

By applying the matrix inversion lemma [8, p.18] to the blockstructured matrix $\left(\breve{\Phi} \breve{\Phi}^{T}\right)^{-1}$ and after some extended matrix manipulations, we arrive at Eq. (3). The details are omitted for brevity. Complete proof can be found in [4]

Note that there is no loss in generality in considering a projection vector $p$ with $\|p\|=1$ (i.e. in the ball $\mathcal{B}$ ), since any non-zero projection vector can be re-scaled to have unit-norm and this does not affect the compressive sensing estimation.

Let $\Theta_{1}$ and $\Theta_{2}$ denote, respectively, the matrices $V V^{T} B \Sigma B^{T} V V^{T}$ and $V V^{T}$ in Lemma 1. In the special case that we use the projection operation to collect a measurement from a single sensor, i.e. the projection vector $p$ contains one 1 and all other elements are zeros, then the result in Lemma 1 says that we should collect from the sensor $i$ if the $\frac{\Theta_{1,(i, i)}}{\Theta_{2,(i, i)}}$ is the biggest, where $\Theta_{1,(i, i)}$ and $\Theta_{2,(i, i)}$ are respectively the $i$-th diagonal elements of $\Theta_{1}$ and $\Theta_{2}$. However, the collection of a measurement from a single sensor in general does not give the maximum information gain or reduction in entropy. By using standard results in matrix theory, the projection vector $p$ that maximises entropy reduction is given by the generalised eigenvector of the matrix pencil $\left(\Theta_{1}, \Theta_{2}\right)$ that corresponds to the largest generalised eignevalue [15]. Although this optimal projection vector can be readily computed, this optimal projection vector generally contains many non-zero elements which means that the energy cost to acquire this optimal projection can be high. Since a WSN has limited energy reserve, it is therefore important to consider both information gain and energy consumption simultaneously.

\section{B. Balancing information gain and energy consumption}

In order to strike a balance between information gain and energy consumption, we propose that in each iteration we choose the projection vector $p \in \mathcal{B}$ to maximise the ratio

$$
Q(p)=\frac{\Delta H(p)}{E(p)}
$$

where $E(p)$ is the minimum energy, measured in terms of the number of wireless transmissions, needed to acquire the projection $p^{T} y$ from the WSN. (Note: Adaptive compressive sensing algorithms in [10], [20] seek to find a $p$ to maximise the information gain $\Delta H(p)$.) In other words, we choose the projection vector that gives the maximum information gain per energy cost needed to acquire it. Although the projection vector that maximises $\Delta H(p)$ can readily be computed, the problem of maximising $Q(p)$ is in general NP-hard.

Theorem 1: Assuming that we measure $E(p)$ by the number of packet transmissions needed to compute the projection $p$ in a WSN, then the optimisation problem $\max _{p \in \mathcal{B}} \frac{\Delta H(p)}{E(p)}$ is NP-hard.

Proof: We will show that the problem is NP-hard for a particular instance of the problem. Consider the case where the WSN is fully connected, then for a given projection vector $p$, the minimum energy $E(p)$ needed to acquire this projection is to use the shortest tour to connect all the sensors whose corresponding projection vector coefficients are non-zero. The energy cost needed is equal to the length of the tour and since the network is fully connected, it is in turn equal to the number of non-zero elements in the projection vector $p$. Let $\operatorname{card}(p)$ denote the number of nonzero elements in $p$. The optimisation problem can equivalently be written as $\max _{k=1, . ., n} \frac{1}{k} \max _{p \in \mathcal{B} ; \operatorname{card}(p)=k} \Delta H(p)$. Since $\log$ is a strictly increasing function, maximising $\Delta H(p)$ is equivalent to maximising $\frac{p^{T} \Theta_{1} p}{p^{T} \Theta_{2} p}$. The optimisation problem $\max _{p \in \mathcal{B} ; \operatorname{card}(p)=k} \frac{p^{T} \Theta_{1} p}{p^{T} \Theta_{2} p}$ is an instance of the sparse Linear Discriminant Analysis which is known to be NP-hard [15]. Hence the theorem.

Since the problem of choosing a projection vector $p$ to maximise $Q(p)$ is NP-hard, we propose a number of heuristics to solve this problem. The aim of these heuristics is to determine a good projection vector, which is equivalent to solving the following two sub-problems: (1) The determination of the locations of the non-zero coefficients of the projection vector, which is in fact a routing problem as we argue in Section II ; (2) The determination of the value of the nonzero coefficients of the projection vector to maximise the 
information gain. However, once the locations of the nonzero coefficients of a projection vector have been determined, the value of the non-zero coefficients can be determined by solving a generalised eigenvector problem. This means that the key problem is to determine to the locations of the non-zero coefficients of the projection vector.

\section{Heuristics}

In this section, we present a number of heuristics, of increasing complexity, to solve the optimisation problem $\max _{p \in \mathcal{B}} \frac{\Delta H(p)}{E(p)}$. In order to simplify the design of the heuristics, we will approximate $\Delta H(p)$ by $\frac{1}{2} \log \left(1+\frac{1}{\hat{\sigma}^{2}} p^{T} \Theta_{1} p\right)$. Note that this is not expected to affect the result much since $\Theta_{2}$ is an idempotent matrix [8]. Note that the problem remains NP-hard even with this approximation.

Algorithm 1 (Sensor with best information gain per energy): In this algorithm, the sink will use a projection to obtain a sensor measurement from a sensor. In other words, the projection vector $p$ is limited to have only one non-zero element. The optimisation problem can be written as $\max _{i=1, \ldots, n} \max _{p_{i}=1 ; p \in \mathcal{B}} \frac{\Delta H(p)}{E(p)}$ which can be solved by computing the value of $\frac{\Delta H(p)}{E(p)}$ for each sensor, where for the $i$-th sensor, $\Delta H(p)=\frac{1}{2} \log \left(1+\Theta_{1,(i, i)}\right)$ where $\Theta_{1,(i, i)}$ is the $(i, i)$-th element in the matrix $\Theta_{1}$ and $E(p)$ is the number of hops to sensor $i$ via the least hop path times 2 .

Algorithm 1 can be implemented by the sink sending a query packet to the chosen sensor which will then return its measurement to the sink. However, this algorithm only uses the sensor measurement of the end-point of the path but it does not use any information from the sensors along the path. It can readily be shown that a projection vector that uses all sensors along a path will always give a higher entropy reduction than a projection vector that uses only a subset of sensors along the same path; moreover, the energy consumption of these two situations are equal, so it will be an advantage to use all the sensors along the path too. Based on this discussion, we propose the following algorithm.

Algorithm 2 (Shortest path with best information gain per energy): Consider a sensor node $i \in V$, let $\mathcal{P}_{i}$ be the set of sensor nodes along the least hop path from the sink to sensor $i$ excluding the sink. We abuse the notation and write $p \in \mathcal{P}_{i}$ if the non-zero elements of the projection vector $p$ correspond only to the sensors in $\mathcal{P}_{i}$. i.e. $p \in \mathcal{P}_{i}$ iff $p_{m}=0 \forall m \notin \mathcal{P}_{i}$. Algorithm 2 chooses the projection vector which maximises $\max _{i=1, \ldots, n} \frac{\max _{p \in \mathcal{P}_{i} ; p \in \mathcal{B}} \Delta H(p)}{2\left|\mathcal{P}_{i}\right|}$.

A shortcoming of Algorithm 2 is that the path calculation does not take the entropy reduction into consideration when it decides which nodes are to be included in the path. In Algorithms 3 and 4, we present two heuristics where the choice of nodes is determined by entropy reduction.

Algorithm 3: Greedy Path The key idea of Algorithm 3 is to find a path with a good ratio of entropy reduction to path length. The following description refers to the symbolic description of the algorithm. This algorithm loops $n$ times where in the $i$-th iteration, we determine a path $P_{i}$ from node $i$ to the sink. After all $P_{i}$ 's $(i=1, \ldots, n)$ have been determined, we choose the projection vector $p$ that maximises

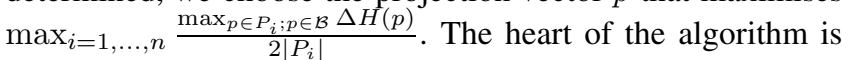
in the for-loop in lines 5-10 where $P_{i}$ is determined. The variable $P_{i}$, which is the set of nodes that are to be included in the path, is initialised to contain the node $i$. Given that node $i$ is $h_{i}$ hops away from the sink, in the first iteration in lines $5-10$, the algorithm finds all neighbours of node $i$ which are $h_{i}-1$ hops away from the sink. These neighbours are put into the set $N_{j}$ (line 6). The algorithm then runs through all the combinations $\{i, v\}$ with $v \in N_{j}$ to see which of them gives the largest reduction in entropy (line 7) and this node is then included in $P_{i}$ (line 8). The process then repeats and in each iteration, a node is added to $P_{i}$ and the nodes in $P_{i}$ become a hop closer to the sink. The output of the algorithm (line 15) is the path to be used where the nodes on the path are stored in $P_{\hat{i}}$ and the corresponding projection vector $\hat{p}$.

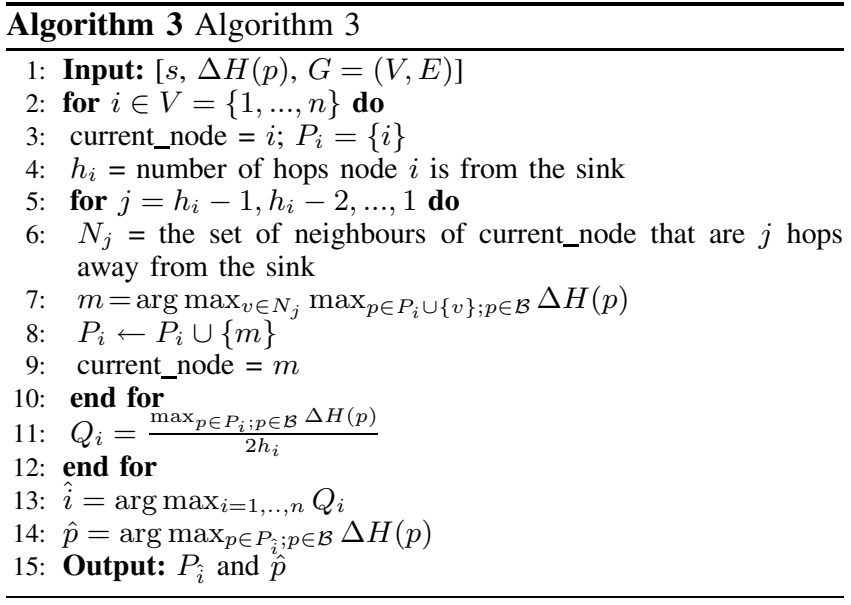

Algorithm 4: Greedy Tour A weakness of Algorithm 3 is that the projection is computed along a path from the sink to node $i$, which means the message will go through the intermediate nodes in the path twice. It is possible to achieve greater reduction in entropy if the nodes in the forward path from the sink to node $i$ are different from those in the reverse path from node $i$ to the sink. Therefore, Algorithm 4 finds a tour through the node $i(i=1, \ldots, n)$ and chooses the tour that gives the best entropy reduction to energy expenditure ratio. The following exposition refers to the symbolic description of Algorithm 4. Algorithm 4 aims to find two node-disjoint paths from node $i$ to the sink if possible. It finds the first path from node $i$ to the sink using Algorithm 3 (line 5 of Algorithm 4). It then runs another for-loop (lines 6-12) to find a nodedisjoint path from node $i$ to the sink. Note that this for-loop is essentially identical to that in lines 5-10 in Algorithm 3 except that it considers only nodes that have not been chosen already in order to achieve node-disjointness (line 7). The rest of the Algorithm 4 is essentially the same as Algorithm 3.

\section{Performance eValuation}

We will illustrate the performance of our algorithms by using simulation as well as data collected from an outdoor WSN. 


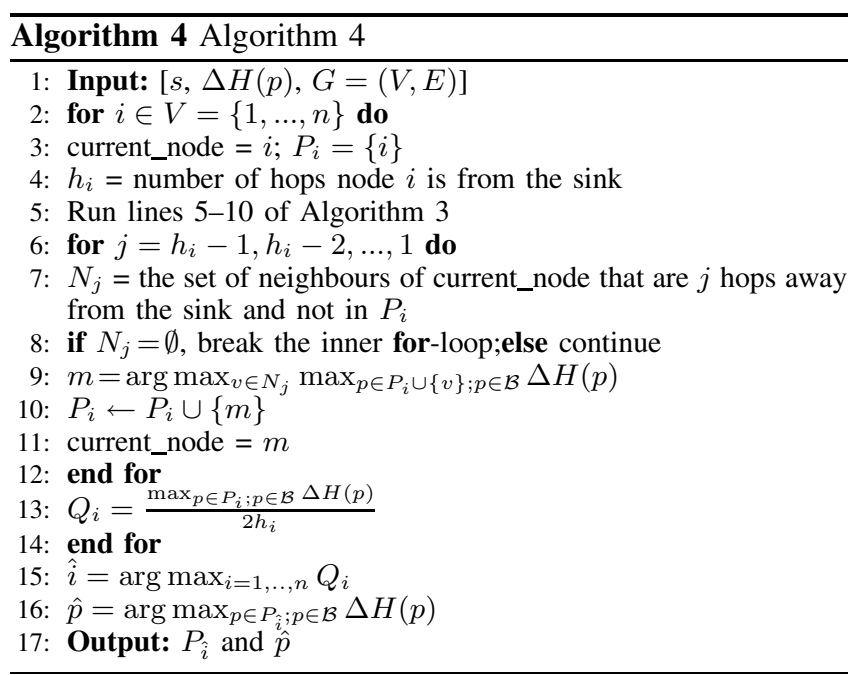

\section{A. Simulation}

We consider a WSN with 256 sensors arranged in a regular square grid in a two-dimensional plane. We assume the sensors are located at $\left(i-8-\frac{1}{2}, j-8-\frac{1}{2}\right)$ for $i, j \in\{1,2, \ldots, 16\}$ on the $(x, y)$-plane. The sink is located at the centre of the square grid at $(0,0)$. Two nodes in the WSN are connected if they are within a distance of 1.5 units of each other. Therefore a node has a maximum of 8 neighbours. We assume that a shortest path tree has already been built in the network.

We generate correlated data on the sensor network using an algorithm similar to the one used in [16]. Let $d_{i j}$ be the distance between sensors $i$ and $j$, then we assume that the correlation of the data between them is given by $\exp \left(-0.5 d_{i j}\right)$. Let $C$ be the resulting correlation matrix. We compute the Cholesky factorisation of $C=L L^{T}$ and generate a realisation of the noise-free data by $L z$ where $z$ is a vector of i.i.d. random numbers. Sensor noise with variance of 0.005 is then added to the noise-free data. The data field can be shown to be compressible in the Discrete Cosine Transform (DCT) [19] basis, see [4] for details.

We apply the generic adaptive compressive sensing algorithm (Algorithm 0) to the data. We assume that in the initial part of the algorithm (in line 1), 80 random sensors return their noisy readings to the sink and 80 additional projections are performed in the loop between lines 2-6. Algorithms 1-4, which are described in Section III-C, are used to determine a good projection vector (line 3 ). In addition, we use the following three algorithms as references:

- Algorithm MaxEnt: The sink uses the projection vector that gives the maximum reduction in entropy, i.e solution of $\max _{p \in \mathcal{B}} \Delta H(p)$. Note that this $p$ is non-sparse, so we expect high energy consumption but good accuracy.

- Algorithm MaxEntNode: The sink asks the sensor that gives maximum reduction in entropy to return its reading.

- Algorithm Random: The sink randomly asks one of the sensors that has not been queried before to return its reading.

We choose two different metrics to measure the performance. For accuracy, we use the relative reconstruction error

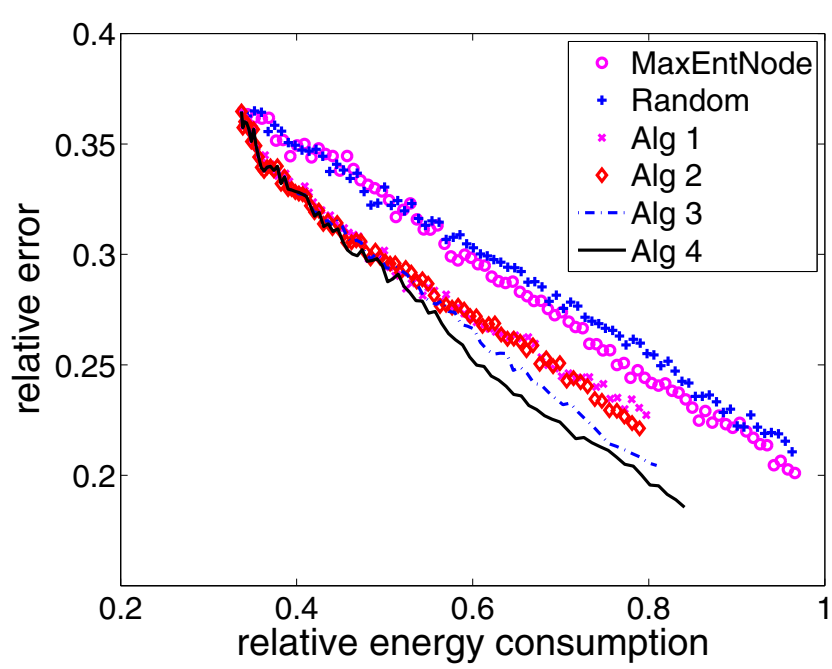

Fig. 2. Performance of Algorithms 1-4 and reference algorithms MaxEntNode and Random.

$\frac{\|x-\hat{x}\|}{\|x\|}$ defined in Section II. For energy, we count the total number of transmissions used up to each iteration. For example, if 50 additional projections have been used, then the total number of transmissions include those coming from the 80 initial sensor readings and the 50 additional projections. Let $J$ represent the number of transmissions then we express our results as $\frac{J}{J_{\text {ref }}}$ where $J_{\text {ref }}$ is the total number of transmissions required by the sensors if they all send their data to the sink, i.e. if $\frac{J}{J_{\text {ref }}} \geq 1$ then the method does no better than the sensor nodes simply return all their data to the sink.

Figure 2 compares the performance of Algorithms 1-4 against that of the reference algorithms MaxEntNode and Random. The results are obtained from the average of 100 simulation experiments. It can be seen that Algorithms 3 and 4 - which make use of a well chosen path or tour to balance accuracy and energy consumption - give the best result in the sense that, for a given energy consumption, these two algorithms give the least relative error. Figure 3 compares the performance of Algorithm 4 with the reference algorithm MaxEnt. It shows that MaxEnt can give a lower relative error after the same number of projections but it also requires much more energy because the optimal projection vector for MaxEnt is non-sparse. In fact, Figure 3 shows that the energy consumption of MaxEnt is worse than simply having the sensors return the data to the sink. This demonstrates that the existing adaptive compressive sensing algorithms cannot be applied directly to WSNs because they do not take energy expenditure to acquire projections into consideration. Note that we have elected to plot only the results for Algorithm 4 in Figure 3 because of the horizontal scale needed to plot the result of MaxEnt means that the results of Algorithms 1-4 will all be cluttered near the left-hand-side of the graph and render them indistinguishable from each other.

\section{B. Outdoor WSN}

We evaluate the performance of our algorithms using the sensor readings collected from the CSIRO sensor deployment 


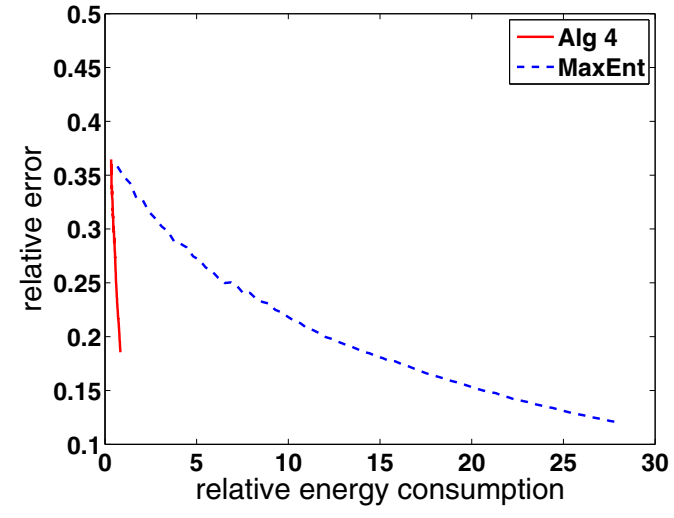

Fig. 3. Performance of Algorithm 4 and reference algorithm MaxEnt.

in Belmont, Queensland, Australia. As shown in Fig. 4 that there are 32 nodes in the Belmont deployment which are arranged in a $4 \times 8$ grid. The sink node is located to the left of this grid. Nodes in this deployment have similar connectivity to those discussed in Sect. IV-A, i.e. each of them can have maximum eight neighbors. We synchronized the nodes and collected one month of temperature data with a sampling interval of one minute. The number of snapshots used is 42,646 .

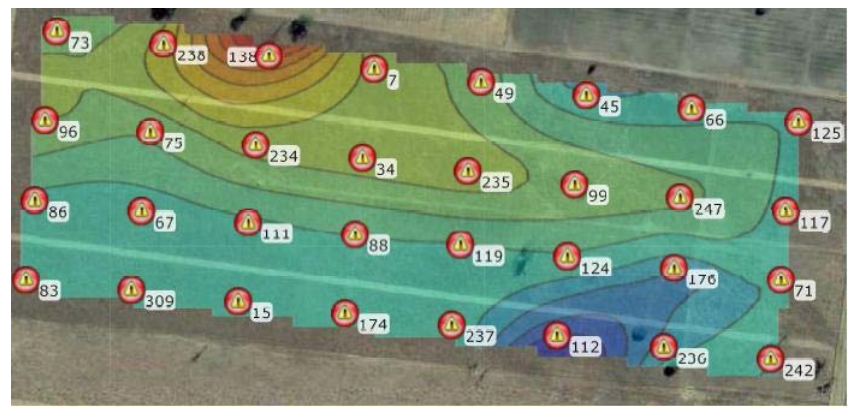

Fig. 4. Sensor arrangement in Belmont, The sink node is neighbor to node 96,86 and 83 .

1) Basis selection for microclimate Data: In order to find an appropriate basis for the temperature signal, we compute its representation in a number of bases, including DCT, Fourier and a number of different wavelets bases (e.g. Haar, Daubechies (D4), Symlets, Coiflets, and Splines etc.). For a given spatial signal and a given basis, we compute the relative reconstruction error between the original signal and its approximation by retaining only the largest $k(k=1,2, \ldots)$ coefficients in that basis. This process is repeated for all the temperature signals collected over the one month period and for all the different bases mentioned above. Fig. 5 shows the results for the DCT, Fourier and D4 bases. (Note that we have presented the results from only the best three bases to avoid cluttering the figure.) We observe that, for the same percentage of coefficients, the representation in the DCT basis gives a lower reconstruction error compared with the other bases.

From the above comparison, we identify that the tempera-

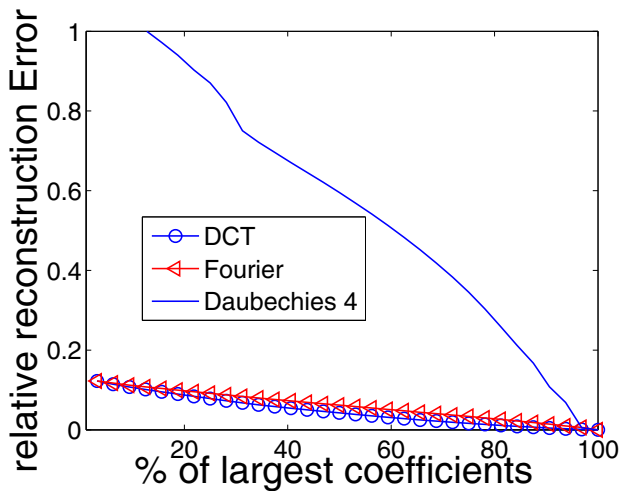

Fig. 5. This figure shows the relative reconstruction error of the temperature signal ( $y$-axis) for a given percentage of largest coefficients that are being retained ( $x$-axis) when the signal is represented in a particular basis.

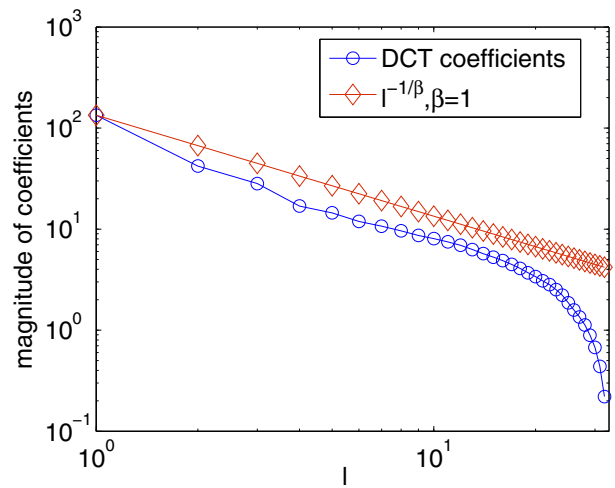

Fig. 6. This figure shows the DCT coefficients of the temperature signal are decaying faster than $\ell^{-1 / \beta}$ with $\beta=1$. In this $(\log -\log )$ plot, the $x$-axis is the index $\ell$ and the $y$-axis is the absolute value of the coefficients.

ture signal has the most compressible representation in the DCT basis. However, a question may be posed here that whether the representation of the temperature signal in the DCT basis satisfies the requirements of a compressible signal discussed in Section II. To answer this question, we plot, in Figure 6, the absolute value of the DCT coefficients in descending order of magnitude along as well as the function $\ell^{-1 / \beta}$ with $\beta=1$ (which appears as a straight line in a $\log -\log$ plot). We find that for $\beta=1$, each of the ordered coefficients decays quicker than $\ell^{-1 / \beta}$. This result implies that the temperature signal is sufficiently compressible in the DCT basis. Compressibility of the temperature signal in the DCT basis can also be found intuitively from Figure 5. This figure shows that it requires only $10 \%$ of the DCT coefficients to keep the relative reconstruction error to within 0.1 .

2) Applying adaptive compressive sensing: We apply the generic adaptive compressive sensing algorithm (Algorithm 0) to the 42,646 snapshots of temperature data using DCT as the basis. We assume that in the initial part of the algorithm (line 1), 10 random sensors return their readings to the sink and 10 additional projections are performed in the loop between lines $2-6$. We use Algorithms 1-4 and MaxEntNode to determine a projection vector. Fig. 7 compares the perfor- 


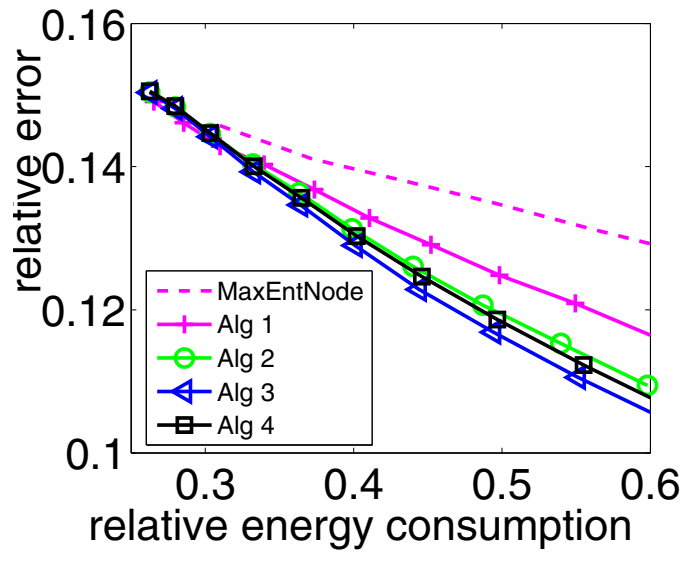

Fig. 7. Reconstruction accuracy and energy consumption trade-off the reconstruction of the temperature signals.

mance of Algorithms $1-4$ against that of the reference algorithm MaxEntNode where the average relative error and average energy consumption are plotted. Similar to the results in Section IV-A, Algorithms 3 and 4 give the least relative error for a given energy expenditure. These two algorithms give $11 \%$ relative error in the estimated data field for an energy saving of $40 \%$.

\section{RELATED WORK}

There is a rich literature on adaptive sampling for WSNs, see e.g. [6], [11], [13], [16]. Our work distinguishes from these earlier works in that it uses adaptive compressive sensing. This means that our focus is on computing a good projection, which is a generalised form of measurements. However, the earlier works use the traditional form of measurement which means that they decide whether to collect sensing data from a specific sensor at a specific time or not.

The paper [1] proposes to compute projections in WSNs by using an additive MAC channel. This work is complementary to ours in the sense that it proposes an alternative method to compute projections. However, [1] does not use adaptation in determining its projection. We expect that the work in [1] can benefit from adaptation. The paper [17] proposes to obtain projections in WSNs via gossiping; however, the paper does not study energy consumption requirement.

Two recent papers [12], [18] studied how compressive sensing can be applied to WSNs taking into account the energy required to obtain the projections from the network. The goal of both of these papers is similar to ours, which is to obtain a sufficiently accurate snapshot of the data field (estimated by using compressive compressing) by spending as little energy as possible to acquire the necessary projections. In particular, both papers [12], [18] demonstrate the important connection between the computation of a projection in WSNs and routing. (See also our discussion in Section II.) An important distinction between our work and the work in [12], [18] is that we use adaptive compressive sensing to iteratively design good projection vectors. This iterative design involves the determination of a good route as well as good projection vector coefficients.

\section{Conclusions}

This paper has proposed a framework to adaptively collect information from a wireless sensor networks using adaptive compressive sensing taking into account both energy consumption and the amount of information in the sensing data. We show that the problem of computing a projection in adaptive compressive sensing that maximises information gain to energy expenditure is NP-hard and we propose a number of heuristics to solve this problem. Our performance evaluation with simulated data and real sensor network data shows that our algorithms give accurate estimation of the unknown data for a given energy expenditure.

Acknowledgment: The authors would like to thank CSIRO for providing the data used in Section IV-B. This research is partially supported by the Australian Research Council Discovery Grant DP0770523.

\section{REFERENCES}

[1] W. Bajwa et al., Joint Source-Channel Communication for Distributed Estimation in Sensor Networks. IEEE Trans Information Theory, 2007.

[2] E. J. Candes and M. Wakin. An introduction to compressive sampling. IEEE Signal Processing Magazine, March 2008.

[3] E. J. Candes et al., Robust uncertainty principles: Exact signal reconstruction from highly incomplete frequency information, IEEE Trans. Inf. Theory, 2006.

[4] C.T. Chou, R. Rana and W. Hu. Cross-layer interactions in energy efficient information collection in wireless sensor networks with adaptive compressive sensing. Technical report ftp://ftp.cse.unsw.edu.au/pub/doc/ papers/UNSW/0909.pdf

[5] V.V. Fedorov. Theory of Optimal Experiments. Academic Press, 1972.

[6] H. Gupta, V. Navda, S. Das and V. Chowdhary. Efficient gathering of correlated data in sensor networks. In ACM Trans. on Sensor Networks, 2008.

[7] J. Haupt, R. D. Nowak: Signal Reconstruction From Noisy Random Projections. IEEE Transactions on Information Theory 52(9): 4036-4048 (2006)

[8] R.A. Horn and C.R. Johnson. Matrix Analysis, Cambridge University Press, 1988.

[9] W. Hu, C. T. Chou, S. Jha, N. Bulusu: Deploying long-lived and costeffective hybrid sensor networks. Ad Hoc Networks 4(6): 749-767 (2006)

[10] S. Ji, Y. Xue, and L. Carin. Bayesian Compressive Sensing. IEEE Trans. Signal Processing, 2008.

[11] J. Kho, A. Rogers, N.R. Jennings. Decentralised control of adaptive sampling in wireless sensor networks. ACM Trans. on Sensor Networks, 2009.

[12] S. Lee et al. Spatially-localized compressed sensing and routing in multihop sensor networks. In Proc. of 3rd Int. Conf. on GeoSensor Networks (GSN 2009), 2009.

[13] C. Liu, K. Wu and J. Pei. An energy-efficient data collection framework for wireless sensor networks by exploiting spatialtemporal correlation. IEEE Trans. on Parallel and Distributed Systems, 2007.

[14] D. J. C. MacKay. Bayesian interpolation. Neural Comp. 4, 1991.

[15] B. Moghaddam, Y. Weiss, and S. Avidan. Generalized spectral bounds for sparse LDA. In Proc. ICML, 2006.

[16] A. Muttreja, A. Raghunathan, S. Ravi and N.K. Jha, Active Learning Driven Data Acquisition for Sensor Networks. Proc. of the 11th IEEE Sym. on Computers and Communications (ISCC'06), 2006.

[17] M. Rabbat et al., Decentralized compression on predistribution via randomized gossiping. Proc. IPSN 2006.

[18] G. Quer et al. On the interplay between routing and signal representation for compressive sensing in wireless sensor networks. In Information Theory and Applications Workshop, 2009.

[19] K. Sayood. Introduction to Data Compression, Morgan Kaufmann, 2000.

[20] M.W. Seeger, Bayesian Inference and Optimal Design for he Sparse Linear Model. Journal of Machine Learning Research, 2008. 